\title{
Erratum: Modeling the quasistatic energy transport between nanoparticles [Phys. Rev. E 92, 062138 (2015)]
}

\author{
George Y. Panasyuk and Kirk L. Yerkes
}

(Received 20 September 2020; published 30 September 2020)

DOI: 10.1103/PhysRevE.102.039902

In Eq. (49), a factor $\epsilon_{v k}$ in the left-hand side was omitted. Corrected, Eq. (49) is

$$
\frac{d}{d t} E_{v}=\sum_{k=1}^{N} \epsilon_{v k} \dot{E}_{v k}=-\left[J_{v}^{(1)}+J_{v}^{(2)}\right]
$$

where

$$
\epsilon_{v k}=\frac{C}{\hat{h}\left(z_{k}\right)}\left[Z_{\nu}\left(z_{k}\right)-A_{v k}^{(2)}(0)\left(2 z_{k}^{2}\right)^{-1}\right]
$$

with $\hat{h}\left(z_{k}\right), Z_{v}\left(z_{k}\right)$, and $A_{v k}^{(p)}(t)$ determined from (23), (28), and (46), respectively; $C=2 \hat{\gamma} D^{2} / \pi$. As one can check, $\epsilon_{\nu k}=$ $O(1)$. Corrected relations (54)-(56) are

$$
\begin{aligned}
& \sum_{k=1}^{N} \epsilon_{1 k} \dot{E}_{1 k}=-\sum_{k=1}^{N}\left[\left(j_{1 k}+j_{11 k}\right) E_{1 k}+j_{12 k} E_{2 k}\right], \\
& \sum_{k=1}^{N} \epsilon_{2 k} \dot{E}_{2 k}=-\sum_{k=1}^{N}\left[\left(j_{2 k}+j_{22 k}\right) E_{2 k}+j_{21 k} E_{1 k}\right],
\end{aligned}
$$

and

$$
\sum_{k=1}^{N}\left(\epsilon_{1 k} \dot{E}_{1 k}+\epsilon_{2 k} \dot{E}_{2 k}\right)=0
$$

Correspondingly, relations (60) and (61) become

$$
\epsilon_{1 k} \dot{E}_{1 k}=R_{1 k} E_{1 k}-R_{2 k} E_{2 k} \equiv-J_{k}
$$

and

$$
\epsilon_{2 k} \dot{E}_{2 k}=R_{2 k} E_{2 k}-R_{1 k} E_{1 k} \equiv J_{k},
$$

where the coefficients $R_{1 k}$ and $R_{2 k}$ are correct and determined from (62) and (63). Finally, due to the corrected Eq. (56) and the mode independence, the relation between $\delta E_{1 k}(t)$ and $\delta E_{2 k}(t)$ becomes $\epsilon_{2 k} \delta E_{2 k}(t)=-\epsilon_{1 k} \delta E_{1 k}(t)$.

The origin of the factors $\epsilon_{v k}$ can be explained as follows (see, also, [1]). Using formulas (A1)-(A5) from the Appendix and relation (38) for $E_{v k}$, one can reduce the expression for

$$
E_{v}=\sum_{i=1}^{N_{v}}\left\langle\frac{p_{v i}^{2}}{2 m_{v i}}+\frac{m_{v i} \omega_{v i}^{2} x_{v i}^{2}}{2}\right\rangle
$$

to

$$
2 E_{v}=\sum_{k} E_{1 k} \sum_{i=1}^{N_{v}}\left(e_{v i}^{k}\right)^{2}+\sum_{k} E_{2 k} z_{k}^{-2} \sum_{i=1}^{N_{v}}\left(w_{v i} e_{v i}^{k}\right)^{2},
$$

where

$$
\sum_{i=1}^{N_{v}}\left(e_{v i}^{k}\right)^{2}=\frac{C}{\hat{h}\left(z_{k}\right)} A_{v k}^{(2)}(0)=\frac{C}{\hat{h}\left(z_{k}\right)} Z_{v}\left(z_{k}\right)
$$

and

$$
\begin{aligned}
\sum_{i=1}^{N_{v}}\left(w_{v i} e_{v i}^{k}\right)^{2} & =-\frac{C}{\hat{h}\left(z_{k}\right)} A_{\nu k}^{(4)}(0) \\
& =\frac{C}{\hat{h}\left(z_{k}\right)}\left[z_{k}^{2} Z_{v}\left(z_{k}\right)-A_{v k}^{(2)}(0)\right] .
\end{aligned}
$$

Thus, after substitution of (4) and (5) into (3), the resulting expression for $E_{v}$ reads

$$
E_{v}=\sum_{k}\left(\epsilon_{1 k} E_{1 k}+\epsilon_{2 k} E_{2 k}\right),
$$

where $\epsilon_{v k}$ are determined from (1). Reducing the left-hand side of (5) using the approach used to derive the approximate expression (29) for $Z_{v}\left(z_{k}\right)$, one can simplify the expression for

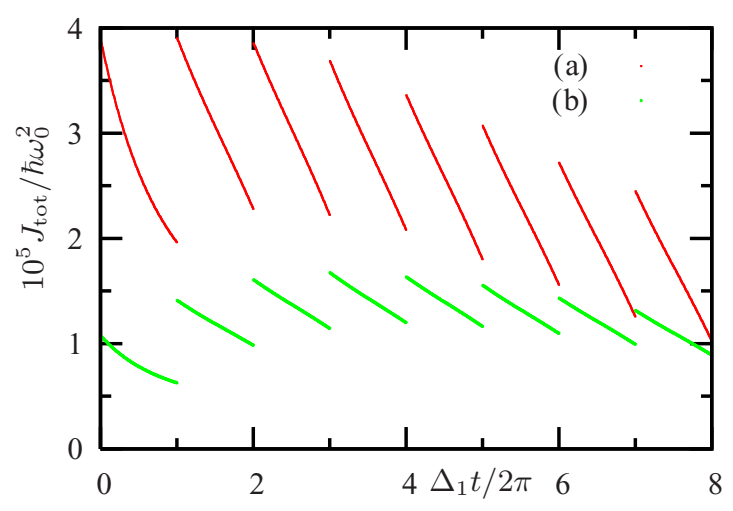

FIG. 7. Time dependences of $J_{\text {tot }}$ between identical nanoparticles when $\hat{\gamma} / \omega_{0}=0.1, D / \omega_{0}=1$, and $\Delta_{1} / \omega_{0}=\Delta_{2} / \omega_{0}=0.001$ and $\alpha=0.01$ (a) $k_{\mathrm{B}} \bar{T} / \hbar w_{0}=0.2$ and (b) $k_{\mathrm{B}} \bar{T} / \hbar w_{0}=0.15$. 


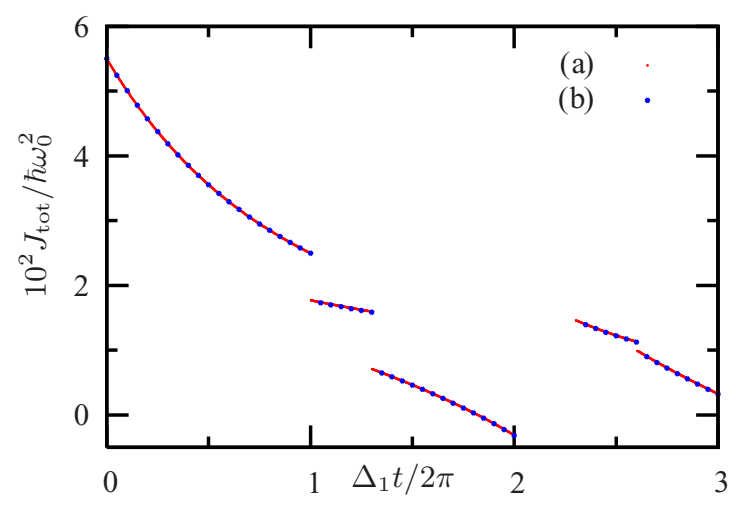

FIG. 8. Time dependences of $J_{\text {tot }}$ between unequal nanoparticles when $\hat{\gamma} / \omega_{0}=0.1, D / \omega_{0}=1$, and $\Delta_{1} / \omega_{0}=0.001, \Delta_{2} / \omega_{0}=$ 0.0013 and $\alpha=0.01$. (a) $k_{\mathrm{B}} \bar{T} / \hbar w_{0}=0.2$ and (b) $k_{\mathrm{B}} \bar{T} / \hbar w_{0}=0.1$.

$\epsilon_{v k}$ to

$$
\epsilon_{\nu k}=\frac{\Delta_{v_{1}} \sin ^{2}\left(\pi z_{k} / \Delta_{\nu_{1}}\right)}{\Delta_{1} \sin ^{2}\left(\pi z_{k} / \Delta_{1}\right)+\Delta_{2} \sin ^{2}\left(\pi z_{k} / \Delta_{2}\right)},
$$

where $v_{1}=1$ if $v=2$, and $v_{1}=2$ if $v=1$. If $\Delta_{1}=\Delta_{2}, \epsilon_{1 k}=$ $\epsilon_{2 k}=1 / 2$.

The main idea underlying the derivations of the coefficients $j_{1 k}, j_{11 k}, j_{12 k}, j_{2 k}, j_{22 k}$, and $j_{21 k}$ is the same as in [1]. It is based on an observation that each time when $t$ passes $t_{n m}$ from (65), the time integrals, such as

$$
\int_{0}^{t} d s g(s) \sin \left(w_{\nu i} s\right) \text { and } \int_{0}^{t} d s g(s) \cos \left(w_{\nu i} s\right),
$$

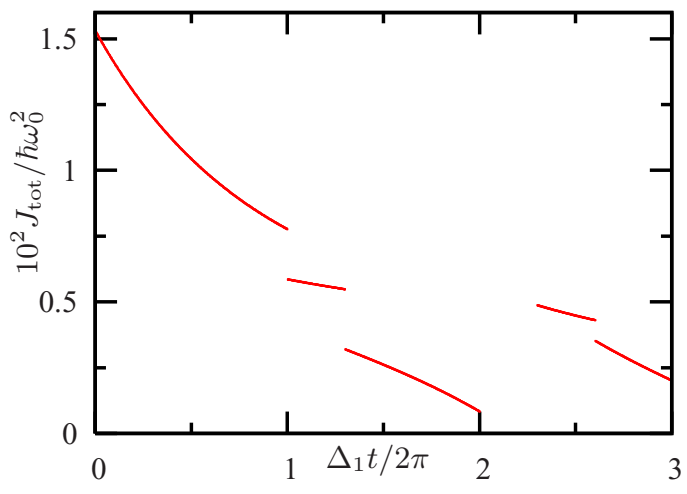

FIG. 9. Time dependences of $J_{\text {tot }}$ between Pt nanoparticles when $\hat{\gamma} / \omega_{0}=0.13, D / \omega_{0}=0.35$, and $\Delta_{1} / \omega_{0}=0.001, \Delta_{2} / \omega_{0}=0.0013$, $k_{\mathrm{B}} \bar{T} / \hbar w_{0}=0.1$, and $\alpha=0.01$.

change their values and stay approximately unchanged until next time moment $t_{n^{\prime} m^{\prime}}$. This happens because the solution kernel $g(t)$ from (22) is noticeably nonzero only at times within time intervals $\left[t_{n m}-\tau, t_{n m}-\tau\right]$ near $t_{n m}$, where $\tau$ is the characteristic microscopic time from (24). Details of this derivation can be found in Appendix B from [1]. In this work, we also developed another way to derive the same coefficients. It is based on the accurate time averaging of expressions (36) and (37). As we found, the produced coefficients essentially coincide with the ones obtained in the way described above. The latter way, however, is preferable because it is much faster than the accurate time averaging.

Although these corrections do not qualitatively change the main results of the article (no revisions of the Abstract and Conclusions are needed), they lead to some modifications of the energy current between the nanoparticles, which are reflected in Figs. 7-9.

[1] G. Y. Panasyuk, K. L. Yerkes, and T. J. Haugan, Phys. Rev. E 99, 032141 (2019). 